\title{
Cell fusion experiments reveal distinctly different association characteristics of cell-surface receptors
}

\author{
Péter Nagy ${ }^{1,2,3, *}$, László Mátyus ${ }^{1, *}$, Attila Jenei ${ }^{1,3, *}$, György Panyi ${ }^{1}$, Sándor Varga ${ }^{4}$, János Matkó ${ }^{1}$, \\ János Szöllösi ${ }^{1}$, Rezső Gáspár ${ }^{1}$, Thomas M. Jovin ${ }^{3}$ and Sándor Damjanovich ${ }^{1, \mp}$ \\ 1 Department of Biophysics and Cell Biology, Medical and Health Science Center, University of Debrecen, Debrecen, Hungary \\ ${ }^{2}$ Cell-biophysical Workgroup of the Hungarian Academy of Sciences, Nagyerdei krt. 98, PO Box 39, H-4012 Debrecen, Hungary \\ ${ }^{3}$ Department of Molecular Biology, Max Planck Institute for Biophysical Chemistry, Am Faßberg 11, D-37077 Göttingen, Germany \\ ${ }^{4}$ Central Research Service Laboratory, Medical and Health Science Center, University of Debrecen, H-4012 Debrecen, Hungary \\ *These authors contributed equally to the work \\ ¥Author for correspondence (e-mail: dami@jaguar.dote.hu) \\ Accepted 26 July 2001 \\ Journal of Cell Science 114, 4063-4071 (2001) (C) The Company of Biologists Ltd
}

\section{SUMMARY}

The existence of small- and large-scale membrane protein clusters, containing dimers, oligomers and hundreds of proteins, respectively, has become widely accepted. However, it is largely unknown whether the internal structure of these formations is dynamic or static. Cell fusion was used to perturb the distribution of existing membrane protein clusters, and to investigate their mobility and associations. Scanning near-field optical microscopy, confocal and electron microscopy were applied to detect the exchange of proteins between large-scale protein clusters, whereas photobleaching fluorescence energy transfer was used to image the redistribution of existing small-scale membrane protein clusters. Largescale clusters of major histocompatibility complex (MHC)I exchanged proteins with each other and with MHC-II clusters. Similarly to MHC-I, large-scale MHC-II clusters were also dynamic. Exchange of components between small-scale protein clusters was not universal: intermixing did not take place in the case of MHC-II homoclusters; however, it was observed for homoclusters of MHC-I and for heteroclusters of MHC-I and MHC-II. These processes required a fluid state of the plasma membrane, and did not depend on endocytosis-mediated recycling of proteins. The redistribution of large-scale MHC-I clusters precedes the intermixing of small-scale clusters of $\mathrm{MHC}$-I indicating a hierarchy in protein association. Investigation of a set of other proteins ( $\alpha$ subunit of the interleukin 2 receptor, CD48 and transferrin receptor) suggested that a large-scale protein cluster usually exchanges components with the same type of clusters. These results offer new insight into processes requiring time-dependent changes in membrane protein interactions.

Key words: Fluorescence resonance energy transfer, FRET, Scanning near-field optical microscopy (SNOM), Lipid rafts, Protein association, Cell fusion

\section{INTRODUCTION}

The classical experiment of Frye and Edidin proved that major histocompatibility complex (MHC) proteins are mobile in the plasma membrane of heterokaryons of human and mouse lymphocytes (Frye and Edidin, 1970). This conclusion was incorporated by Singer and Nicolson into their fluid mosaic membrane model of 1972, which emphasized the random distribution and free lateral mobility of membrane proteins (Singer and Nicolson, 1972). However, experimental evidence has accumulated suggesting a locally restricted mobility of proteins in the lipid bilayer (Edidin, 1997). In addition, co-capping experiments and intermolecular distance determinations by fluorescence resonance energy transfer (FRET) measurements are indicative of a nonrandom distribution of proteins in the plasma membrane (Damjanovich et al., 1997b). Several important cell-surface proteins, e.g. MHC-I and MHC-II (Matkó et al., 1994; Szöllosi et al., 1996) and the subunits of the interleukin-2 receptor (IL-2R) (Damjanovich et al., 1997a), engage in homo- and heteroassociations; FRET experiments have indicated that these proteins are within 2-10 $\mathrm{nm}$ of each other (Damjanovich et al., 1997b). Such associations are referred to as small-scale clusters. Beyond these associations single particle tracking (Edidin et al., 1991), electron microscopy, scanning force microscopy (Damjanovich et al., 1995) and scanning near-field optical microscopy (SNOM) (Hwang et al., 1998; Nagy et al., 1999) reveal a second hierarchical level of protein associations. These so-called large-scale clusters are several hundred nanometers in diameter containing tens to thousands of proteins. In an earlier work we predicted such a nonrandom molecular distribution of transmembrane proteins, based on the preferential accommodation of genetically determined $\alpha$ helical transmembrane protein domains into distinct membrane microdomains (Damjanovich et al., 1981). This hypothesis is consonant with the raft hypothesis, which states that sphingomyelin- and cholesterol-containing lipid islands (rafts) may accommodate a selected set of glycosylphosphatidylinositol (GPI)-linked and transmembrane proteins (Simons and Ikonen, 1997).

The Singer-Nicolson membrane model postulated a random and continuous redistribution of membrane proteins in the unstructured lipid environment, but did not address the extent to which the protein clusters described above are 
dynamic. The Frye-Edidin experiment proved the redistribution of proteins in the plasma membrane on the micrometer-scale, but could not distinguish between the following two scenarios: first, the composition of protein clusters at the plasma membrane is constant, and intermixing of proteins results from movement of intact large-scale protein clusters; second, proteins are exchanged between clusters by dynamic association-dissociation events. The second possibility will be referred to as dynamic composition. Here we present experimental data revisiting the Frye-Edidin system using the FRET and SNOM techniques, which can explore both hierarchical levels of receptor codistribution patterns. We have found that dynamism is the rule, rather than the exception, in the composition of membrane protein clusters. However, MHC-II forms such tight small-scale associations that exchange of proteins is completely blocked. Free exchange of components between clusters takes place only in the fluid state of the plasma membrane, and does not depend on protein recycling. By investigating a series of raft and nonraft proteins we found that preformed large-scale homoclusters of proteins are not statically associated, and exchange of components between like clusters is usually observed.

\section{MATERIALS AND METHODS}

\section{Cells and antibodies}

The B lymphoblastoid cell line, JY, and the IL-2-dependent T leukemic cell line, Kit 225 K6, were cultured in RPMI-1640 medium with $10 \%$ FCS. The medium for Kit225 K6 was supplemented with $20 \mathrm{U} / \mathrm{ml}$ (20 pM) IL-2 every second day. W6/32 and L-243 Fabs were used to label MHC-I and MHC-II, respectively. Fab fragments of MEM75 and MEM102 antibodies, kindly provided by Vaclav Horejsi (Institute of Molecular Genetics, Academy of Sciences, Prague, Czech Republic) were used to label transferrin receptor (TrfR) and CD48, respectively. The $\alpha$ subunit of the IL-2R (IL-2R $\alpha$ ) was labeled with anti-Tac Fab (T. A. Waldmann, NIH, Bethesda, MD).

\section{Cell labeling and fusion}

To characterize the small- and large-scale homoassociation of a protein, one cell sample was labeled with Fab against the protein labeled with a fluorescent dye (fluorescein or Cy3), whereas another cell sample was labeled with the same type of Fab tagged with a different dye (rhodamine, Cy3, Cy5). In the investigation of the heteroassociation of two different proteins the two cell samples were separately labeled with distinct Fabs against the proteins. The Fabs were tagged with different fluorophores as mentioned above. Capital $\mathrm{F}$ and $\mathrm{R}$ refer to fluoresceinated and rhodaminated Fabs, respectively. In the case of labeling with colloidal gold for electron microscopy cells were incubated with primary unlabeled antibodies. This was followed by incubation with secondary antibodies conjugated to gold beads: two samples were separately labeled with 10 and $30 \mathrm{~nm}$ beads (Amersham Pharmacia, Buckinghamshire, UK).

A pellet of the mixture containing approximately 5-10 million cells was fused with $200 \mu \mathrm{l} 50 \%$ polyethylene glycol (PEG, MW 8000) at room temperature (Hui et al., 1985). Fused cells were incubated, and samples were taken every 20 minutes and fixed in $1 \%$ formaldehyde for fluorescence microscopic FRET measurements. For SNOM, cells were dropped on poly-L-lysine-coated glass coverslips, fixed on the glass with $4 \%$ formaldehyde and dehydrated in an ethanol series on ice. For electron microscopy cells were spread on poly-L-lysinecoated Formwar grids, dehydrated in an ethanol series and air-dried from ether.

\section{Fluorescence microscopy}

An inverted fluorescence microscope (Axiovert 135TV, Zeiss) was used for photobleaching FRET measurements (Jovin and Arndt Jovin, 1989). To characterize the association of proteins in nonfused membranes the donor photobleaching time constant was determined on donor-acceptor double-labeled samples and on donor-only-labeled samples on a pixel-by-pixel basis. To follow the redistribution of proteins after membrane fusion the donor photobleaching time constant was measured on fused cells; identification was based on the presence of both fluorescence labels on them. This value was compared to the donor photobleaching time constant determined on fused, donor-only labeled cells. Polyethylene glycol applied to induce cell fusion did not alter the spectral characteristics of the fluorescent dyes. Histograms of the time constants were generated from 10,00030,000 pixels on $10-15$ different cells. The average FRET efficiency was calculated using histogram means of the photobleaching time constants.

\section{Scanning near-field optical microscopy}

The SNOM was an add-on to a Nanoscope-IIIa scanning probe system (Digital Instruments, Santa Barbara, CA) (Kirsch et al., 1998). Simultaneous excitation and detection of fluorescence emission were performed with an uncoated optical fiber tip. Excitation of fluorescein and Cy5 was with the $488 \mathrm{~nm}$ and $647 \mathrm{~nm}$ lines, respectively, of a Performa argon-krypton laser (Spectra Physics, Mountain View, CA), whereas excitation of rhodamine was with the $543 \mathrm{~nm}$ line of a He-Ne laser (LHGP 0101, Research Electro Optics, Boulder, CO). Suitable excitation, dichroic and emission filters were selected to minimize spillover between fluorescence channels. SNOM images of double-labeled cells were processed in Scil-Image (Technical University of Amsterdam, The Netherlands). Low-frequency background was removed using either a Fourier transformation based method (Hwang et al., 1998) or top-hat transformation (Glasbey and Horgan, 1995). High-frequency noise was removed either with low-pass filtering or by a morphology operation (opening) (Glasbey and Horgan, 1995). Cluster and background pixels were discriminated with the entropy threshold algorithm of Scil-Image (segmentation). To calculate the area of overlap between clusters of different proteins, segmented images (in which digit 1 and 0 corresponds to cluster and background pixels, respectively) recorded in different fluorescence channels were multiplied with each other. Fractional overlap was calculated by dividing the area of overlap by the area of one of the membrane protein clusters.

\section{Confocal microscopy}

A Zeiss LSM410 confocal laser scanning microscope system was used to image nonfixed and nondehydrated cells. Excitation of rhodamine-labeled and fluorescein-labeled monoclonal antibodies was carried out with the $543 \mathrm{~nm}$ line of a HeNe laser and with the $488 \mathrm{~nm}$ line of an Ar ion laser, respectively. Separation of the exciting and emitted photons was achieved with a $488 / 543 \mathrm{~nm}$ dichroic mirror. Rhodamine and fluorescein emissions were discriminated with a 560 $\mathrm{nm}$ dichroic mirror. Detection of rhodamine and fluorescein emissions was with a $570 \mathrm{~nm}$ long-pass filter and with a 510-535 nm band-pass filter, respectively. Analysis of confocal images was done in a manner similar to that used for SNOM images.

\section{Electron microscopy}

Gold bead-labeled cells were imaged in a JEOL electron microscope (JEM $100 \mathrm{~B}$ microscope operated at $80 \mathrm{kV}$ ) on the periphery and thinner parts of cells, where transparency allowed good contrast. Correlation between the distribution of $10 \mathrm{~nm}$ and $30 \mathrm{~nm}$ gold beads was evaluated as follows. A grid with a cell size of about $300 \times 300$ $\mathrm{nm}$ was placed on electron microscopic images, and the number of 10 $\mathrm{nm}$ and $30 \mathrm{~nm}$ gold beads was counted. The correlation coefficient between the number of $10 \mathrm{~nm}$ beads per cell and the number of 30 
$\mathrm{nm}$ beads per cell was calculated. The significance of correlation was tested using analysis of variance (ANOVA).

\section{Investigation of the internalization of Fab}

Internalization of cell-surface-bound Fab fragments was followed by the acid-wash method (Baulida and Carpenter, 1997). Samples taken from fluorescent Fab-labeled cells were divided into two parts. One of the samples was treated with a $\mathrm{pH} 2.8$ buffer $(0.2 \mathrm{M}$ Na-acetate, $0.5 \mathrm{M} \mathrm{NaCl}$ ) for 3 minutes, and its fluorescence intensity was compared to that of the untreated sample. We proved that $\mathrm{pH} 2.8$ reliably removes about $95 \%$ of cell-surface-bound fluorescent ligands, and leaves only the intracellular part of fluorescence. The fluorescence intensity was measured on a Becton Dickinson FacsStar Plus flow cytometer.

\section{Investigation of the recycling of Fab}

To decide whether Fab fragments recycle with their respective antigen the following experiments were carried out. Two parallel samples were prepared. Both samples were preincubated with $10 \mu \mathrm{g} / \mathrm{ml}$ cycloheximide for 1 hour, and all procedures were carried out in the presence of cycloheximide to block protein synthesis. Sample 1 was labeled with a fluoresceinated Fab fragment, and incubated at $37^{\circ} \mathrm{C}$. Sample 2 was incubated without Fab labeling. Samples were taken at regular intervals, and those from sample 2 were labeled with fluoresceinated Fab. The membrane fluorescence of these samples was calculated by subtracting the fluorescence intensity after acid wash from the total intensity. If the Fab fragments recycle with the antigen, all the antigens in the plasma membrane will be Fab labeled even after incubation at $37^{\circ} \mathrm{C}$. Therefore the membrane fluorescence intensity of sample 1 will be the same as that of sample 2 . If only the antigen without Fab recycles, some portion of the antigens in the membrane will not carry a fluorescent label in sample 1, therefore the membrane fluorescence intensity of sample 1 will be lower than that of sample 2.

\section{RESULTS}

\section{MHC-I forms dynamic homoclusters on the surface of JY lymphoblasts}

Homoclusters of MHC-I molecules have previously been identified on B cells (Matkó et al., 1994). To confirm or refute the dynamic composition of protein clusters, we fused JY cells separately labeled with fluoresceinated W6/32 (anti-MHC-I heavy chain) and rhodaminated W6/32 Fab, and followed the kinetics of intermixing of these labels with FRET and SNOM measurements (Fig. 1). FRET is usually considered to correlate with small-scale associations of membrane proteins (Damjanovich et al., 1997b), whereas SNOM has proven efficient in identifying large protein clusters (several hundred nanometers in diameter) (Hwang et al., 1998; Nagy et al., 1999). Regular fluorescence microscopy indicated that there was a clear separation of the fluorescein and rhodamine signals immediately after cell fusion, but subsequently MHC-I became evenly distributed in the fused plasma membrane at $37^{\circ} \mathrm{C}$. In addition, the SNOM images clearly showed that the composition of large-scale clusters was also dynamic (Fig. 2C); the overlap of red and green clusters reached $67 \%$ after 80 minutes (Fig. 1). Because fixation and dehydration, which are necessary steps in sample preparation for near-field microscopy, may artificially cause protein clustering, we checked for the presence of similar clusters on the surface of non-dehydrated, non-fixed cells with confocal microscopy. The average size and dynamic behavior of large-scale protein

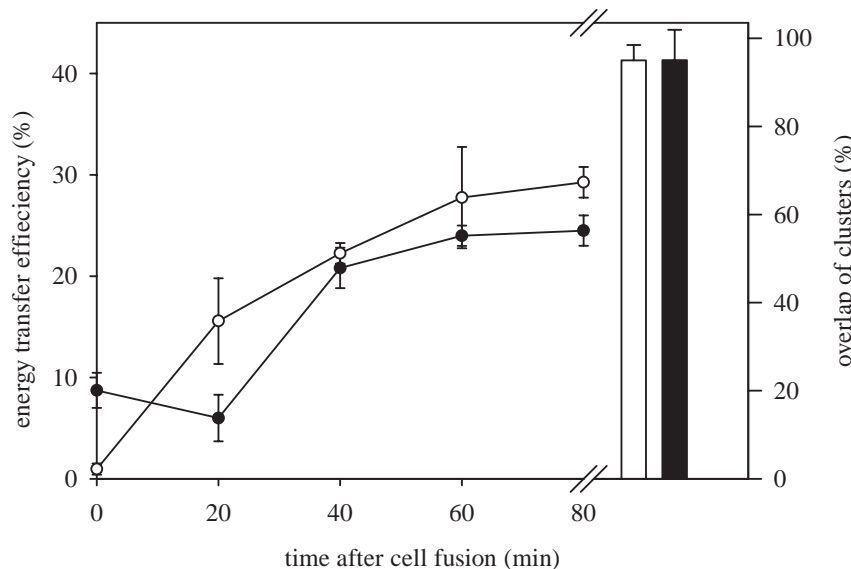

Fig. 1. Intermixing of small- and large-scale protein clusters follows a different time course. Two samples of JY cells were labeled separately with fluoresceinated and rhodaminated anti-MHC-I Fabs (W6/32), respectively. The two separately labeled samples were subsequently fused. Temporal changes in FRET efficiency between fluoresceinated and rhodaminated W6/32 Fabs $(-$ and overlap of fluorescein and rhodamine clusters $(\bigcirc)$ were measured. The filled bar on the right displays the FRET efficiency measured between fluoresceinated W6/32 and rhodaminated W6/32 on a doublelabeled, non-fused JY cell sample, whereas the white bar shows the overlap of fluoresceinated W6/32 and rhodaminated W6/32 clusters on similarly labeled cells. Results are mean \pm s.e.m.

clusters identified with confocal microscopy (Fig. 2G,H) was similar to those observed with near-field microscopy. To examine further the dynamic composition of protein associations we measured the FRET efficiency (characterizing direct protein-protein interactions) between fluoresceinated W6/32 and rhodaminated W6/32 Fabs. Although there was a lag of about 20 minutes in the FRET curve behind the SNOM curve, the FRET efficiency reached $60 \%$ of the value observed with non-fused double-labeled cells (Fig. 1). This result clearly indicates the occurrence of dynamic rearrangements in the composition of small-scale MHC-I clusters.

\section{Translational membrane diffusion is required for the intermixing of MHC-I clusters after cell fusion}

To define whether the fluid state of the membrane is required for the above-defined hierarchical redistribution of proteins, the same experiments were carried out with the fused cells kept on ice. Neither SNOM nor FRET experiments revealed any significant degree of protein redistribution (Fig. 2D; Fig. 3), implying that the dynamic composition of protein clusters depends on membrane diffusion. However, it was necessary to demonstrate that intermixing of proteins takes place in the plasma membrane and not during a process of endocytosismediated recycling. Neither brefeldin A, monensin nor aluminum fluoride $\left(\mathrm{AlF}_{3}\right)$ blocked internalization of MHC-I (data not shown). In the case of both the FRET and SNOM experiments, the emission was collected from plasmamembrane-bound fluorescent Fab fragments. Therefore, it was sufficient to establish that Fab fragments did not recycle with their protein targets, implying that fluorescent Fab fragments detected in the plasma membrane had not undergone recycling. In control experiments we established that fluorescent Fab did not recycle with the antigen (data not shown). We conclude 


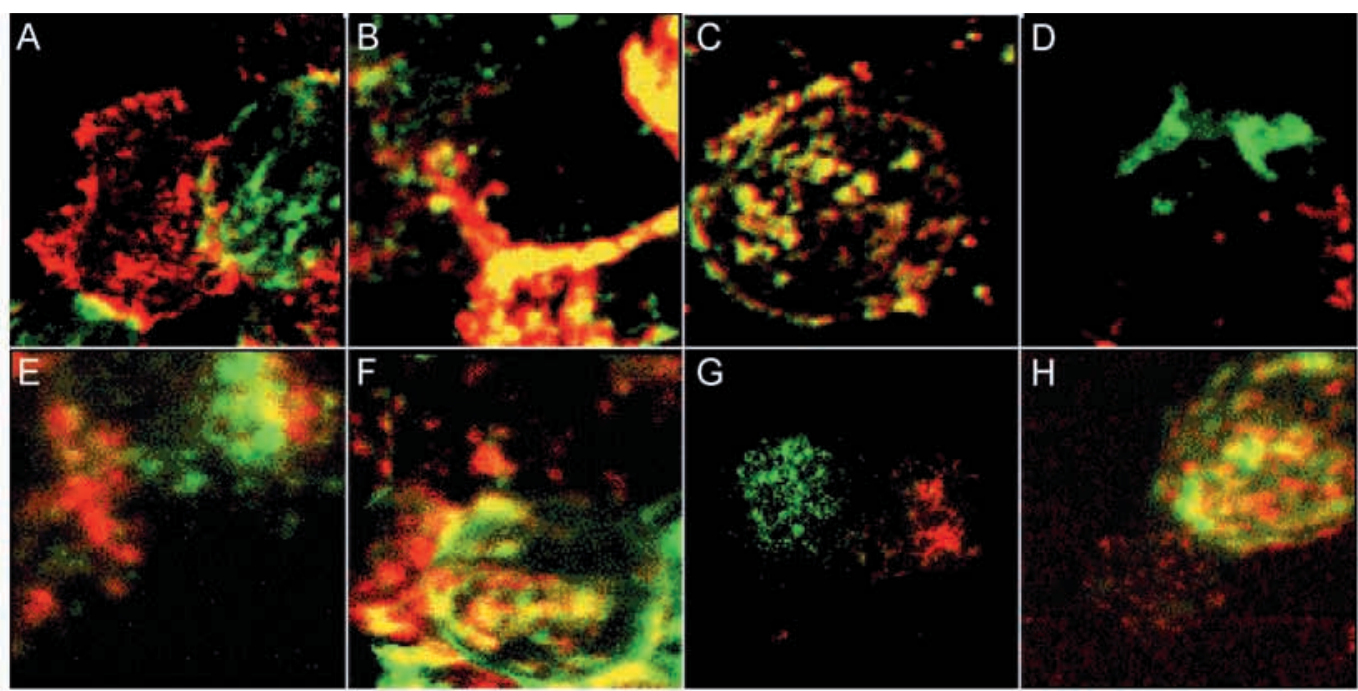

Fig. 2. Scanning near-field optical and confocal microscopical images of PEG-fused cells. Cells were separately labeled with two different Fabs tagged with different fluorescent dyes. These samples were subsequently fused, and incubated at $37^{\circ} \mathrm{C}$, except for $\mathrm{D}$ where incubation was on ice. Intermixing of the fluorescence in the two different channels was monitored using SNOM (A-F) and confocal (G-H) microscopy. The two images collected in the two fluorescence channels were superimposed on each other. The two signals are displayed in green and red, respectively. (A) Kit225 K6 cells, Cy3-IL-2R (green), Cy5-IL-2R (red), 0 minutes after fusion. (B) Kit225 K6 cells, Cy3-IL-2R (green), Cy5IL-2R (red), 80 minutes after fusion. (C) JY cells, F-MHC-I (green), R-MHC-I (red), 80 minutes after fusion. (D) JY cells, F-MHC-I (green), R-MHC-I (red), 80 minutes after fusion, incubation on ice. (E) Kit225 K6 cells, Cy3-CD48 (green), Cy5-CD48 (red), 80 minutes after fusion. (F) Kit225 K6 cells, Cy3-TrfR (green), Cy5-TrfR (red), 80 minutes after fusion. (G) JY cells, F-MHC-I (green), R-MHC-I (red), 0 minutes after fusion, (H) JY cells, F-MHC-I (green), R-MHC-I (red), 80 minutes after fusion. Image size: A-F, $15 \times 15 \mu \mathrm{m}$; G-H, $20 \times 20 \mu \mathrm{m}$.

that dissociation and re-association of small-scale protein complexes and reformation of large-scale associations took place in the plasma membrane. Recycling of MHC-I proteinbound Fab fragments was partially blocked by $\mathrm{AlF}_{3}$ (data not shown). Thus, the fact that $\mathrm{AlF}_{3}$ did not alter the responses detected by either FRET or SNOM confirmed that the observed phenomena occurred exclusively in the plasma membrane.

\section{MHC-II forms static small-scale homoassociation on the surface of JY cells}

It has been shown previously that MHC-II proteins also form homoassociations in the plasma membrane of JY cells (Szöllősi et al., 1996). We investigated whether complexes of MHC-II proteins behave similarly to that of MHC-I. Two samples of JY cells were separately labeled with F-L243 and R-L243 Fab (against MHC-II), and subsequently fused with each other. According to the SNOM experiments large-scale homoclusters of MHC-II were as dynamic as MHC-I clusters (Fig. 3). By contrast, the FRET measurements revealed a strikingly dissimilar behavior of MHC-II in that there was no increase in the FRET values between F-L243 and R-L243 (Fig. 3 ), indicating the absence of dissociation and re-association of MHC-II molecules.

\section{Intermixing of MHC-I and MHC-II protein clusters is observed after fusion of JY cells}

Having characterized the homoclustering of MHC-I and MHCII, we proceeded to an investigation of their heteroassociation on JY cells. Fusion of cells separately labeled with F-L243 and R-W6/32 showed that both small- and large-scale heteroclusters of MHC-I and MHC-II proteins were dynamic; both the FRET efficiency between F-L243 and R-W6/32 Fab fragments and the overlap of MHC-I clusters with those of MHC-II increased after cell fusion (Fig. 3). The above experiments on JY cells proved that clusters of MHC proteins exchange components with each other in the plasma membrane.

\section{Investigation of the dynamic properties of MHC-I and IL-2R clusters on the surface of Kit225 K6 cells}

After the characterization of the dynamic properties of MHC protein associations on a B cell line, we extended the studies to a T-cell line (Kit225 K6). Both large- and small-scale homoassociation of MHC-I displayed the same type of dynamic behavior on $\mathrm{T}$ cells as on the B cell line (Fig. 4). We then turned our attention to the IL-2R, as it fulfills an essential function in $\mathrm{T}$ cells, and protein association is known to be equally important in the case of this receptor (Damjanovich et al., 1997a; Vereb et al., 2000). We separately labeled Kit225 K6 cells with F-anti Tac and R-anti Tac Fab (against the $\alpha$ subunit of IL-2R), and fused them with PEG. SNOM imaging of fused cells revealed that large-scale homoclusters of IL-2R $\alpha$ subunit intermixed with each other after cell fusion (Fig. 2A,B; Fig. 4). In accordance with previous results (Damjanovich et al., 1997a), the small-scale homoassociation of the IL-2R $\alpha$ subunit was low on Kit225 K6 cells. However, not even this low level of FRET between F-anti Tac and R-anti Tac reappeared after fusion of Kit225 K6 cells separately labeled with the Fabs (Fig. 4). The Fab used in our experiments to label the $\alpha$ subunit of IL-2R partly recycled with the receptor after internalization. Therefore, it was necessary to prove that endocytosis and recycling had no influence on either of the FRET or SNOM results. Treatment of Kit225 K6 cells with $\mathrm{AlF}_{3}$ completely blocked internalization of Fab-labeled IL- 

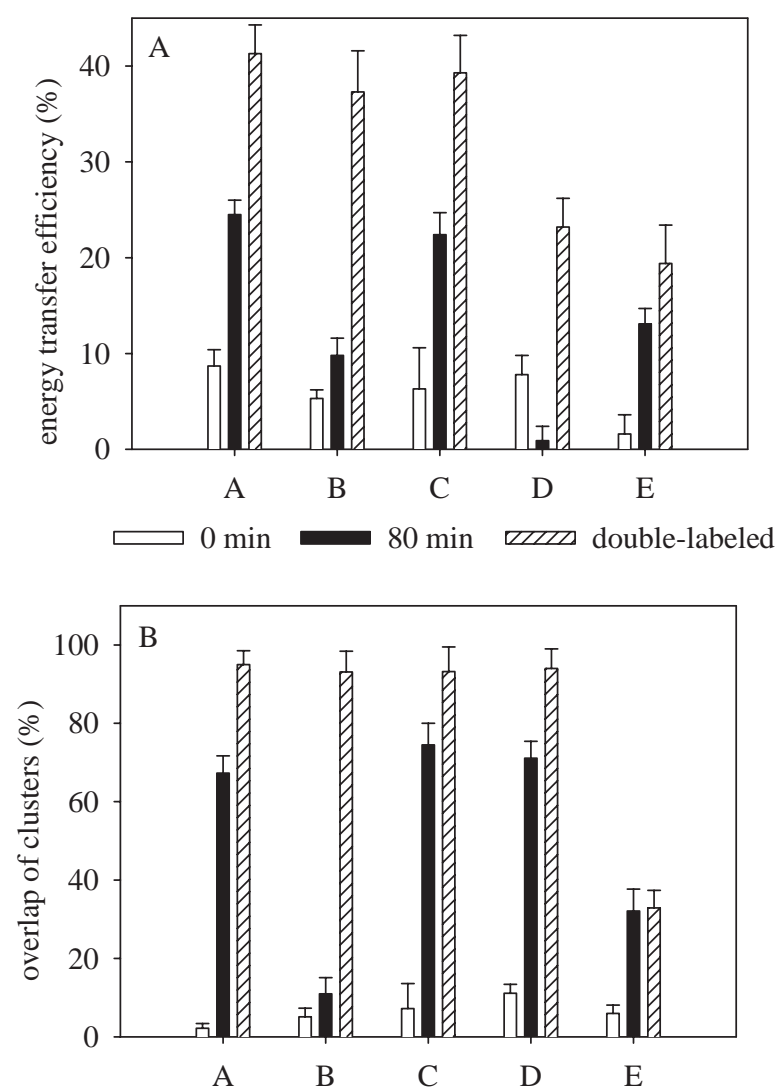

Fig. 3. Intermixing of both small- and large-scale clusters of MHC-I and MHC-II proteins in the plasma membrane of JY cells points to their dynamic nature. Two samples of JY cells were separately labeled with antibodies tagged with different fluorescent labels. (For epitopes labeled by the antibodies see text below.) These samples were subsequently fused with each other using PEG. Cells were then incubated at $37^{\circ} \mathrm{C}$ in all the cases except where it is indicated. The FRET efficiency characterizing small-scale clustering of proteins was determined using the photobleacheing FRET approach 0 and 80 minutes after cell fusion (white and black columns, respectively, in A). Overlap of large-scale clusters was determined in a SNOM (B). When the two proteins labeled by the antibodies were different, overlapping cluster areas were normalized by dividing the doublecolored area with the area of either of the protein clusters. Because the procedures yielded the same results within experimental error, only the normalization with the area of the first protein cluster is presented. As positive controls samples were labeled with antibodies against both proteins (left-hatched columns), and FRET efficiency and overlap percentage was determined similarly. Results are mean \pm s.e.m. Cell-surface receptor pairs were as follows: (A) MHC-I and MHC-I, (B) MHC-I and MHC-I, incubation on ice, (C) MHC-I and MHC-I in the presence of $\mathrm{AlF}_{3}$, (D) MHC-II and MHC-II, (E) MHC-I and MHC-II.

2R $\alpha$ (Fig. 5). Because $\mathrm{AlF}_{3}$ had no effect on either the SNOM or the FRET curves, we conclude that intermixing of largescale clusters of IL-2R $\alpha$ was independent of receptor recycling, and the lack of an increase in the FRET efficiency was similarly independent of endocytosis-mediated recycling.

\section{Both raft and non-raft proteins form dynamic clusters on Kit225 K6 cells}

Some membrane proteins taking part in T-cell activation (e.g. $\mathrm{CD} 3$ ) are known to be associated with lipid rafts, whereas
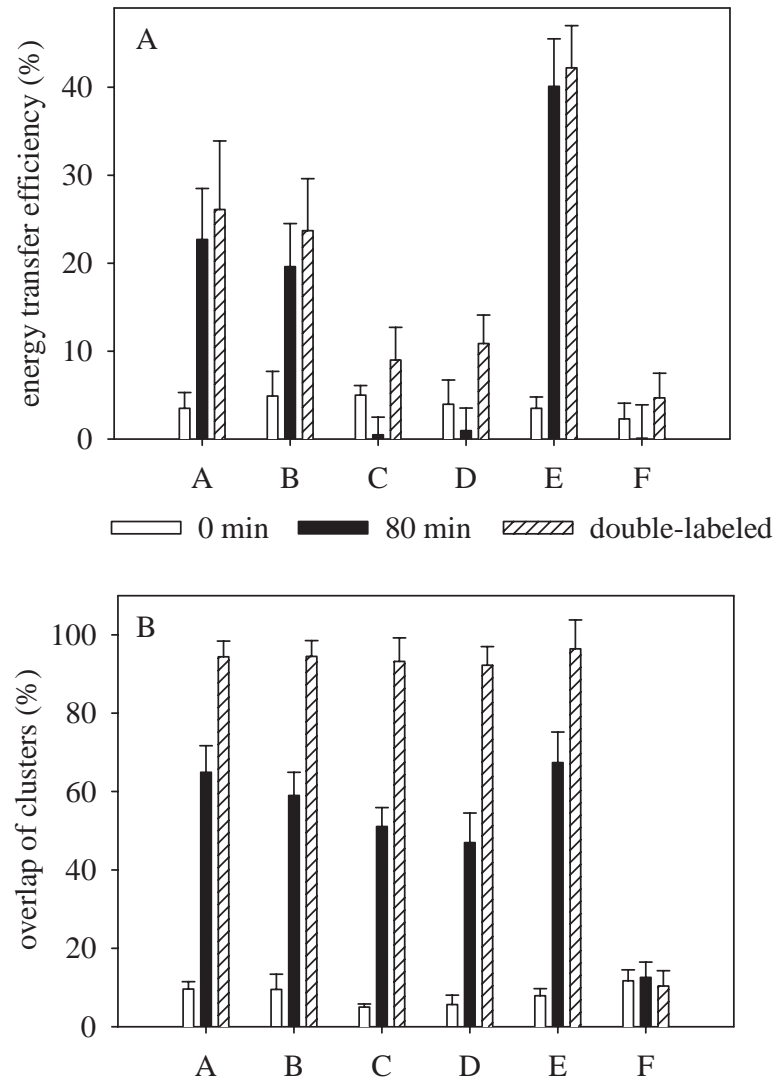

Fig. 4. Raft and nonraft proteins home to their native cluster environment after PEG-induced fusion of Kit225 K6 human T cells. Cells were labeled and analyzed using the same procedures as those described in the legend to Fig. 3. Results are mean \pm s.e.m. A and B refer to data from FRET and SNOM experiments, respectively. Cellsurface receptor pairs were as follows: (A) CD48 and CD48,

(B) TrfR and TrfR, (C) IL-2R $\alpha$ subunit and IL-2R $\alpha$ subunit, (D) IL2 receptor $\alpha$ subunit and IL-2R receptor $\alpha$ subunit in the presence of $\mathrm{AlF}_{3}$, (E) MHC-I and MHC-I, (F) CD48 and TrfR.

others (e.g. CD45) are excluded from these microdomains (Janes et al., 1999). Because aggregation of lipid rafts and temporal changes in lipid raft composition play a key role in T-cell activation (Janes et al., 1999), investigation of their dynamic properties was also of importance. We chose CD48, a GPI-anchored protein, to check the dynamic properties of rafts. First, we investigated the homoclustering of CD48. We separately labeled two samples of Kit225 K6 cells with F-MEM102 and Cy3-MEM102 for FRET measurements, and with Cy3-MEM102 and Cy5-MEM102 for SNOM experiments. The Cy3-Cy5 pair proved to be easier to image in the SNOM, but a dye with a reasonably fast photobleaching is required for photobleaching FRET measurements. Therefore, the fluorescein-Cy3 pair was selected for the FRET experiments. We observed a significant intermixing of largescale CD48 clusters with each other after cell fusion (Fig. 2E; Fig. 4). In addition, FRET between CD48 proteins increased after fusion of cells separately labeled with donor- and acceptor-tagged anti-CD48 Fab fragments (Fig. 4). These results indicate that GPI-anchored protein components of lipid rafts form dynamic small- and large-scale clusters.

The TrfR is known to be excluded from lipid rafts (Harder 


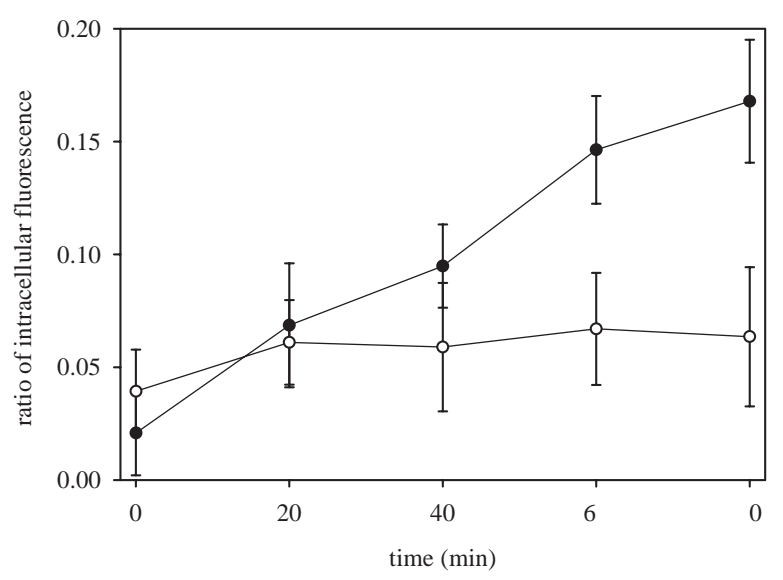

Fig. 5. Internalization of IL-2R is blocked by $\mathrm{AlF}_{3}$. Kit225 K6 cells were labeled with anti-Tac fab against the IL-2R. Cells were incubated in the absence (control sample, - ) or in the presence $(\bigcirc)$ of $\mathrm{AlF}_{3}$ at $37^{\circ} \mathrm{C}$. Samples were taken every 20 minutes, and the ratio of intracellular fluorescence was determined with the acid-wash method as described in Materials and Methods. Results are mean \pm s.e.m.

et al., 1998). Thus, investigation of its clusters was expected to provide information about the dynamic properties of non-raft proteins. Cells separately labeled with F-MEM75 and Cy3MEM75, or Cy3-MEM75 and Cy5-MEM75 (against the TrfR) were fused. The FRET efficiency between F-MEM75 and RMEM75 significantly increased after cell fusion, and largescale homoclusters of TrfR gradually intermixed with each other in 80 minutes after cell fusion (Fig. 4).

We observed an essentially complete segregation of $\operatorname{TrfR}$ from clusters of CD48 on double-labeled non-fused Kit225 K6 cells (Fig. 4). Neither FRET nor SNOM experiments revealed any kind of intermixing between CD48 and TrfR after cell fusion (Fig. 4), in accordance with expectation and indicating that the miscibility of different proteins is restricted by their preferences for distinct lipid environments.

\section{Electron microscopy confirms dynamic large-scale clustering of IL-2R}

To corroborate the SNOM results we used electron microscopy as an independent imaging modality. It has been shown previously that electron microscopy of colloidal gold-labeled cells can reveal protein clustering with an average cluster size of several hundred nanometers (Damjanovich et al., 1995). The IL-2R was labeled with 10 and $30 \mathrm{~nm}$ colloidal gold in two separate samples, which were subsequently fused with PEG. The two different-sized gold beads showed a clear separation at 0 minutes after fusion (Fig. 6A), although there was a minor intermixing of the beads close to the fusion pore. Intermixing of the different gold beads was complete by 80 minutes as indicated by an image taken of a part of a cell far from the fusion pore: no gradient of the concentration of the different gold beads was visible. In addition, the close apposition $(<100 \mathrm{~nm})$ of the two different gold beads indicates that the composition of single protein clusters dynamically changed after cell fusion (Fig. 6B). This distribution is similar to what was found on non-fused cells labeled with both 10 and $30 \mathrm{~nm}$ colloidal gold (Fig. 6C). We determined the correlation coefficient between the distribution of 10 and $30 \mathrm{~nm}$ gold beads. No significant correlation was found on cells immediately after fusion $(\mathrm{C}=0.06, P>0.05)$, whereas strong correlation was observed on double-labeled cells $(\mathrm{C}=0.85$, $P<0.01)$ and on fused cells 80 minutes after fusion $(\mathrm{C}=0.83$, $P<0.05)$. In the determination of gold bead densities $300 \mathrm{~nm}$ wide squares were placed on the image, and the number of beads was counted within them. The size of the squares was chosen so that it matches the characteristic diameter of largescale protein clusters. The fact that significant correlation was observed between the distribution of 10 and $30 \mathrm{~nm}$ gold beads using $300 \mathrm{~nm}$ squares 80 minutes after fusion implies that 10 and $30 \mathrm{~nm}$ beads can get as close as $\sim 300 \mathrm{~nm}$ to each other. This can only occur if proteins labeled with $10 \mathrm{~nm}$ gold beads are exchanged with proteins labeled with $30 \mathrm{~nm}$ beads between clusters. In control experiments PEG treatment of colloidal gold-labeled cells did not lead to detachment of the gold beads from the cell surface (data not shown).

\section{DISCUSSION}

The Singer-Nicolson fluid mosaic membrane model (Singer and Nicolson, 1972) emphasized that free diffusion prevails in the translational motion of membrane components. Although not strictly stipulated in the original concept, the notion of random distribution of membrane proteins thoroughly pervaded concepts about the structure of the cell membrane, as non-random distribution would be expected to be linked to restricted diffusion (Jacobson and Dietrich, 1999). In the past 10-15 years experimental evidence has accumulated, clearly indicating that barriers exist and hinder free lateral diffusion of proteins (Edidin et al., 1991; Wade et al., 1989). Coprecipitation and FRET measurements proved that shortrange order is not uncommon among membrane proteins (Damjanovich et al., 1997a; Matkó et al., 1994). More recently advanced microscopic techniques extended these findings to indicate that long-range order is also general in the cell membrane (Hwang et al., 1998; Nagy et al., 1999). Fluorescence microscopy, used in the Frye-Edidin experiment, cannot resolve the dynamic changes within these small- and large-scale associations, i.e. it is not possible to decide whether proteins engaged in small-scale protein associations can dissociate and reassociate in the plasma membrane. We carried out FRET experiments to answer the above question. Similarly, the intermixing of labeled membrane areas may be the consequence of intermixing of static large-scale protein clusters (their internal composition does not change, they do not exchange components with each other), or alternatively the composition of these large-scale clusters may also undergo dynamic changes. We carried out SNOM, confocal and electron microscopic experiments to answer this question. From the above three SNOM was our major imaging approach. Electron microscopy is labor-intensive. The resolving power of SNOM is better than that of confocal microscopy, and SNOM is an inherently surface imaging device rendering the imaging of cell membranes relatively straightforward. Imaging of curved (i.e. not horizontal) cell membranes is difficult with confocal microscopy, because reconstruction of the membrane surface is necessary, and the poor axial resolution of confocal microscopy deteriorates the overall resolving power.

We investigated the homo- and heteroclustering of several 


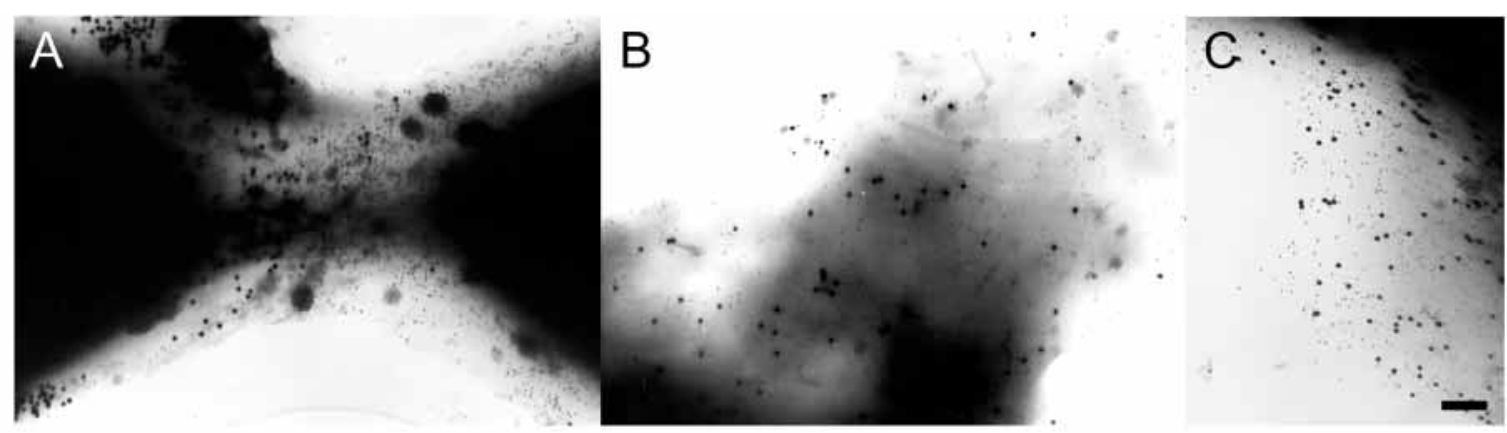

Fig. 6. Colloidal gold labeling of the $\alpha$ subunit of IL-2R reveals receptor intermixing after cell fusion. Kit 225 K6 cells were labeled with antiTac antibodies against the IL-2R $\alpha$ subunit. Two samples of these cells were separately labeled with 10 and $30 \mathrm{~nm}$ colloidal gold beads, and fused with PEG. Samples were taken at 0 minutes (A) and 80 minutes (B) after cell fusion. (C) Nonfused Kit225 K6 cells labeled with both 10 and $30 \mathrm{~nm}$ colloidal gold beads. Bar, $200 \mathrm{~nm}$.

membrane proteins. The patches in the SNOM images were so obvious that no further analysis was necessary to conclude that membrane proteins are clustered on the scale of several hundred nanometers. However, the presence of FRET does not necessarily mean that small-scale protein association is present, because it was shown that a large concentration of randomly distributed molecules also results in FRET (Kenworthy and Edidin, 1998). However, the assumption that FRET is due to and is proportional to clustering is supported by two arguments. First, in some cases no or very low FRET was measured for proteins present at cell surface densities comparable to those showing significant FRET. The lack of FRET between MHC-II proteins after cell fusion and the very low FRET between IL-2R $\alpha$ imply, that this cell surface density of proteins was too low to result in significant FRET by random association. Second, FRET efficiencies, which we considered significant, are higher than expected for a random distribution. Previous calculation also showed that this cell-surface density of proteins is unlikely to result in significant FRET, if the proteins are randomly distributed in the membrane (Trón et al., 1984).

Clustering of MHC-I is well characterized and is known to have important roles in antigen presentation (Damjanovich et al., 1995; Matkó et al., 1994; Valitutti et al., 1995). We found that both small- and large-scale homoassociations of MHC-I are dynamic; i.e. their composition changes after insertion into the endoplasmic reticulum during protein synthesis. There was a delay of around 20 minutes in the FRET curve compared to the SNOM curve during which no increase of FRET took place, whereas cluster overlap increased. This result implies a hierarchy in the association of MHC-I; an initial phase during which intermixing of large-scale clusters takes place followed by direct protein reassociation. This conclusion hinges upon the validity of the assumption that FRET is indicative of molecular interactions. According to the reasoning in the previous paragraph we are certain that this is indeed the case. The existence of two hierarchical levels in the homoassociation of MHC-I has been reported previously (Damjanovich et al., 1995). Our current experiments provide evidence that this hierarchy is also reflected in the dynamic rearrangements taking place in these clusters.

Large-scale homoclusters of MHC-II were as dynamic as those of MHC-I. However, according to the FRET experiments small-scale clustering of MHC-II was static; MHC-II proteins did not swap partners in their homoassociated state. In fact, the FRET values between F-L243 and R-L243 actually decreased after cell fusion, an observation one may attribute to a dilution effect; that is, the probability of accidental encounters between donor-acceptor pairs gradually diminishes as the local membrane concentrations of both donor- and acceptor-labeled antigens decrease during their random redistribution in the larger plasma membrane of the fused cell. The reason for the lack of exchange of proteins between small-scale clusters of MHC-II is unknown. There are indications that MHC-II proteins form dimers, whereas MHC-I can form complexes of five to ten molecules (L. Bene, personal communication). It may be argued that the cohesive force in a dimer is stronger than in a larger complex, inhibiting the escape of MHC-II proteins from association. However, the translational diffusion constant of MHC-I is slightly higher than that of MHC-II (Wade et al., 1989). In addition, because MHC-II contains two transmembrane domains as opposed to the single transmembrane segment of MHC-I, it is plausible that the rotational diffusion constant of MHC-II is also lower than that of MHC-I, thereby inhibiting an optimal alignment for oligomer formation. The fact that FRET efficiency did not increase after cell fusion in all cases implies that cell fusion per se does not disrupt membrane integrity to the extent that nonspecific associations of proteins form spontaneously.

To obtain a deeper insight into the dynamic properties of membrane protein clusters we investigated the behavior of heteroassociations of MHC-I and MHC-II on JY cells. Both small- and large-scale heteroclusters of MHC-I and MHC-II showed dynamic behavior. One may envisage a situation in which overlapping large-scale clusters of MHC-I and MHC-II contain dynamic small-scale homoclusters of MHC-I and static small-scale MHC-II clusters. Although the small-scale homoclusters of MHC-II are not dynamic, they engage in dynamic small-scale association with MHC-I.

We extended our investigations to the IL-2R, which is essential in clonal expansion of antigen-stimulated $\mathrm{T}$ cells (Morris and Waldmann, 2000). Large-scale homoclusters of the $\alpha$ subunit of IL-2R exchanged components with each other in the plasma membrane of fused cells. Because the small-scale homoassociation of IL- $2 \mathrm{R} \alpha$ was very low in non-fused Kit225K6 cells, the failure to detect significant FRET after cell fusion may be due to experimental error or surface dilution of the label. However, one may suppose that only a low 
concentration of IL-2R $\alpha$ homoassociations is present in the membrane, and these are static, i.e. they do not exchange components with each other.

Lipid rafts are membrane microdomains, which serve as signal transduction platforms for GPI-anchored and transmembrane proteins (Ilangumaran et al., 1999). Their protein and lipid composition is different from the rest of the plasma membrane. We chose CD48 and TrfR as representatives of proteins included in and excluded from rafts, respectively. Because there was no difference in the behavior of these two proteins, we concluded that the lipid microenvironment, on its own, does not determine the dynamic properties of protein associations. CD48 and other GPI-anchored proteins are usually thought to be clustered (Varma and Mayor, 1998), but views questioning this contention also exist (Kenworthy et al., 2000). Our results do not favor any of these models, but support that molecular encounters between CD48 proteins, be they the result of density-dependent association or specific interactions, are not tight enough to inhibit the escape of proteins from the association. Clustering of TrfR is thought to be maintained by coated pits (Iacopetta et al., 1988) and the continuous endocytosis and recycling of TrfR (Gheber and Edidin, 1999). The concentration of TrfR in coated pits and the possible clustering of CD48 in a separate membrane microdomain may explain the lack of FRET between these proteins. Our results indicate that 'forces' resulting in protein clustering, whether they are related to membrane trafficking or to microdomain formation, do not generally inhibit the dynamic exchange of protein components between protein clusters, if the clusters reside in the same type of microdomain.

The fluid mosaic membrane model postulated a random diffusion of membrane proteins on the resolution scale of the light microscope. In our current experiments we have extended this model to include the dynamic exchange of protein components between small-scale (nanometer-sized) and large-scale (micrometer-sized) clusters of some proteins, but it has to be stressed that dynamism is not a universal property of small-scale protein associations. The factors determining whether a small-scale protein cluster is dynamic or static are largely unknown. Our model includes two important concepts: concentration of membrane proteins in large-scale clusters, and diffusion of protein components between clusters residing in the same type of lipid environment. Diffusion of proteins in the membrane without clustering is unlikely to generate a high enough local concentration of signaling molecules sufficient for efficient cell stimulation (e.g. T-cell activation). However, the existence of static protein clusters cannot be reconciled with the rapid redistribution of proteins often observed during some physiological processes. These processes require dynamic clusters. Future studies will address how the dynamic vs. static clustering of proteins influence the functioning of cellmembrane-related processes.

This work was supported by research grants F022725, F034487, F035251, T23873, T30411, T23835 and T30399 from the Hungarian Academy of Sciences, ETT 460/2000 from the Hungarian Ministry of Health and by the Max Planck Society. A.J. was the recipient of a long-term fellowship from the European Molecular Biology Organization (EMBO).

\section{REFERENCES}

Baulida, J. and Carpenter, G. (1997). Heregulin degradation in the absence of rapid receptor-mediated internalization. Exp. Cell Res. 232, 167-172.

Damjanovich, S., Somogyi, B. and Trón, L. (1981). Macromolecular dynamics and information transfer. In Neural Communication and Control (ed. G. Székely, E. Lábos and S. Damjanovich), pp. 9-21. New York: Pergamon Press.

Damjanovich, S., Vereb, G., Schaper, A., Jenei, A., Matkó, J., Starink, J. P., Fox, G. Q., Arndt Jovin, D. J. and Jovin, T. M. (1995). Structural hierarchy in the clustering of HLA class I molecules in the plasma membrane of human lymphoblastoid cells. Proc. Natl. Acad. Sci. USA 92, 1122-1126.

Damjanovich, S., Bene, L., Matkó, J., Alileche, A., Goldman, C. K., Sharrow, S. and Waldmann, T. A. (1997a). Preassembly of interleukin 2 (IL-2) receptor subunits on resting Kit $225 \mathrm{~K} 6 \mathrm{~T}$ cells and their modulation by IL-2, IL-7, and IL-15: a fluorescence resonance energy transfer study. Proc. Natl. Acad. Sci. USA 94, 13134-13139.

Damjanovich, S., Gáspár, R., Jr and Pieri, C. (1997b). Dynamic receptor superstructures at the plasma membrane. Q. Rev. Biophys. 30, 67-106.

Edidin, M. (1997). Lipid microdomains in cell surface membranes. Curr. Opin. Struct. Biol. 7, 528-532.

Edidin, M., Kuo, S. C. and Sheetz, M. P. (1991). Lateral movements of membrane glycoproteins restricted by dynamic cytoplasmic barriers. Science 254, 1379-1382.

Frye, L. D. and Edidin, M. (1970). The rapid intermixing of cell surface antigens after formation of mouse-human heterokaryons. J. Cell Sci. 7, 319335 .

Gheber, L. A. and Edidin, M. (1999). A model for membrane patchiness: lateral diffusion in the presence of barriers and vesicle traffic. Biophys. $J$. 77, 3163-3175.

Glasbey, C. A. and Horgan, G. W. (1995). Mathematical morphology. In Image Analysis for the Biological Sciences (ed. C. A. Glasbey and G. W. Horgan), pp. 125-152, John Wiley \& Sons, New York.

Harder, T., Scheiffele, P., Verkade, P. and Simons, K. (1998). Lipid domain structure of the plasma membrane revealed by patching of membrane components. J. Cell Biol. 141, 929-942.

Hui, S. W., Isac, T., Boni, L. T. and Sen, A. (1985). Action of polyethylene glycol on the fusion of human erythrocyte membranes. J. Membr. Biol. 84, 137-146.

Hwang, J., Gheber, L. A., Margolis, L. and Edidin, M. (1998). Domains in cell plasma membranes investigated by near-field scanning optical microscopy. Biophys. J. 74, 2184-2190.

Iacopetta, B. J., Rothenberger, S. and Kuhn, L. C. (1988). A role for the cytoplasmic domain in transferrin receptor sorting and coated pit formation during endocytosis. Cell 54, 485-489.

Ilangumaran, S., Arni, S., van Echten-Deckert, G., Borisch, B. and Hoessli, D. C. (1999). Microdomain-dependent regulation of Lck and Fyn protein-tyrosine kinases in T lymphocyte plasma membranes. Mol. Biol. Cell 10, 891-905.

Jacobson, K. and Dietrich, C. (1999). Looking at lipid rafts? Trends Cell Biol. 9, 87-91.

Janes, P. W., Ley, S. C. and Magee, A. I. (1999). Aggregation of lipid rafts accompanies signaling via the T cell antigen receptor. J. Cell. Biol. 147, 447-461.

Jovin, T. M. and Arndt Jovin, D. J. (1989). FRET microscopy: Digital imaging of fluorescence resonance energy transfer. Application in cell biology. In Cell Structure and Function by Microspectrofluorometry (ed. E. Kohen and J. G. Hirschberg), pp. 99-117. San Diego, CA: Academic Press.

Kenworthy, A. K. and Edidin, M. (1998). Distribution of a glycosylphosphatidylinositol-anchored protein at the apical surface of MDCK cells examined at a resolution of $<100$ A using imaging fluorescence resonance energy tranfer. J. Cell. Biol. 142, 69-84.

Kenworthy, A. K., Petranova, N. and Edidin, M. (2000). High-resolution FRET microscopy of cholera toxin B-subunit and GPI-anchored proteins in cell plasma membranes. Mol. Biol. Cell 11, 1645-1655.

Kirsch, A. K., Meyer, C. K., Huesmann, H., Mobius, D. and Jovin, T. M. (1998). Fluorescence SNOM of domain-structures of LB films containing electron-transfer systems. Ultramicroscopy 71, 295-302.

Matkó, J., Bushkin, Y., Wei, T. and Edidin, M. (1994). Clustering of class I HLA molecules on the surfaces of activated and transformed human cells. J. Immunol. 152, 3353-3360. 
Morris, J. C. and Waldmann, T. A. (2000). Advances in interleukin 2 receptor targeted treatment. Ann. Rheum. Dis. 59, i109-i114.

Nagy, P., Jenei, A., Kirsch, A. K., Szöllosi, J., Damjanovich, S. and Jovin, T. M. (1999). Activation-dependent clustering of the erbB2 receptor tyrosine kinase detected by scanning near-field optical microscopy. J. Cell Sci. 112, 1733-1741.

Simons, K. and Ikonen, E. (1997). Functional rafts in cell membranes. Nature 387, 569-572.

Singer, S. J. and Nicolson, G. L. (1972). The fluid mosaic model of the structure of cell membranes. Science 175, 720-731.

Szöllősi, J., Horejsi, V., Bene, L., Angelisova, P. and Damjanovich, S. (1996). Supramolecular complexes of MHC class I, MHC class II, CD20, and tetraspan molecules (CD53, CD81, and CD82) at the surface of a B cell line JY. J. Immunol. 157, 2939-2946.

Trón, L., Szöllősi, J., Damjanovich, S., Helliwell, S. H., Arndt Jovin, D. J. and Jovin, T. M. (1984). Flow cytometric measurement of fluorescence resonance energy transfer on cell surfaces. Quantitative evaluation of the transfer efficiency on a cell-by-cell basis. Biophys. J. 45, 939-946.

Valitutti, S., Muller, S., Cella, M., Padovan, E. and Lanzavecchia, A. (1995). Serial triggering of many T-cell receptors by a few peptide-MHC complexes. Nature 375, 148-151.

Varma, R. and Mayor, S. (1998). GPI-anchored proteins are organized in submicron domains at the cell surface. Nature 394, 798-801.

Vereb, G., Matkó, J., Vámosi, G., Ibrahim, S. M., Magyar, E., Varga, S., Jenei, A., Gáspár, R., Jr, Waldmann, T. A. and Damjanovich, S. (2000). Cholesterol-dependent clustering of IL-2Ralpha and its colocalization with HLA and CD48 on T lymphoma cells suggest their functional association with lipid rafts. Proc. Natl. Acad. Sci. USA 97, 6013-6018.

Wade, W. F., Freed, J. H. and Edidin, M. (1989). Translational diffusion of class II major histocompatibility complex molecules is constrained by their cytoplasmic domains. J. Cell Biol. 109, 3325-3331. 